\title{
Recent advances and current perspectives in treatment of Alzheimer's disease
}

\section{Khare Ekta $^{1 \text { \& }{ }^{\text {}} \text {, Fatima Zeeshan }}{ }^{2}$}

Received: 31.03.2020

Revised: 06.05.2020

Accepted: 22.05.2020

\begin{abstract}
Dementia is a disorder which is associated with disruption of cerebral neurons, resulting in its characteristic symptomatology. Acetylcholine neurotransmitter is found to be significant for processing memory and learning. However it is diminished in both concentration and function in patients with Alzheimer disease. Nootropics are the drugs which is used to improve memory and learning by acting as AChEI (Acetyl cholineesterase inhibitors). Cognitive enhancers include drugs interacting with receptors (e.g. NMDA receptor antagonist: memantine), Enzymes (e.g. AChE inhibitors: tacrine, donepezil, galantamine), Antioxidants (e.g. resveratrol, curcumin, and acetyl-L-carnitine), Metal chelators (e.g. calcium and zinc chelator: DP-b99), Vaccines, Monoclonal antibodies (e.g. A beta-Amyloid: solanezumab under Phase III clinical trial). Apart from the pharmacological approaches, supplementation of a healthy diet and healthy physical \& mental lifestyle impact cognitive research in the future. There is no remedy for AD. Contemporary treatments just relive the behaviourial symptoms. Treatment centers around making a superior personal satisfaction for the individuals with Alzheimer infection. As of late, undifferentiated cell innovation (stem cell technology), and Nanotechnology has given new bits of knowledge into the treatment of Alzheimer's disease. In this review, we talk about current indicative medicines and future difficulties for new potential illness altering treatments.
\end{abstract}

Keywords: Acetylcholine, AChE inhibitors, Amyloid $\beta$ protein, Antioxidant, Challenges for the development of Antialzheimer's drug, Nanomaterials, tau ( $\tau$ ) protein

\section{Introduction}

A neurological disorder is the biggest problem in the world in which human affects worldwide. Alzheimer's disease (AD), is a unique neurodegenerative disorder which is depicted by the loss of cerebrum work that changes the cholinergic areas of the central nervous system related with subjective capacity, and spatial mindfulness (Barbosa, 2014). Alzheimer disease is the sixth most rudimental reason for death on the planet serious. The impact of Alzheimer's disease is currently estimated to increase either directly or indirectly medical \& social services. (Ernst, 1997). Many steps have been executed during the advancement of small molecule for curing AD. Tacrine was the primary anti-AD drug which was endorsed by the Food Drug Administration (FDA) in 1993 as an Acetyl-cholinesterase (AChE)

\section{Author's Address}

${ }^{I}$ Department of Pharmaceutical Chemistry, Faculty of Pharmacy, ITM University, Gwalior (M.P.), India P.O. Box: 474001

${ }^{2}$ Amity Institute of Pharmacy, Amity University, Lucknow (U.P.), India

E-mail.: ekta.sop@itmuniversity.ac.in inhibitor (Crismon, 1994). Other Acetylcholinesterase (AChE) inhibitor such as donepezil, rivastigmine and galantamine were consecutively endorsed by the FDA throughout the following not many years (Bezerra, 2016). Nevertheless, the present treatment systems of these promoted medications can just mitigate the side effects of Alzheimer disease, however not stop or inverse the movement of ailment (Fan, 2014) and influence a portion of the populace after the age of 60 . With the proceeding with increment in the frequency of $A D$, it is required to significantly increase in the following 50 years in older people. The situation shows up progressively despite other issue that the particular reasons for $\mathrm{AD}$ are still inadequately comprehended, different endeavors have been centered around focusing on Amyloid- $\beta$ peptide (A $\beta)$, Tau protein, Monoamine Oxidase (MAO), $\beta$ site amyloid Precursor Protein-cutting off Enzyme 1 (BACE-1), free radicals and metal particles $\left(\mathrm{Fe}^{2+}\right.$, $\mathrm{Cu}^{2+}, \mathrm{Zn}^{2+}$ ) (Ernst, 1997). Alzheimer disease represents the most gigantic neglected requirement for clinical science and research. The current 


\section{Khare and Fatima}

medications can not, at this point satisfy treatment needs. The refinement of multi-targetted drugs has become a well known region of study. An extending number of joined manufactured mixes and characteristic items with their subordinates have been represented over the span of the latest couple of years as multi-utilitarian administrators to be potential prescription contender for AD. Acetylcholinesterase (AChE) and Butyrylcholinesterase $(\mathrm{BuChE})$ inhibitors are the basic medications in the therapy of Alzheimer's disease. It was exhibited that the hindrance of AChE may prompt an expansion in the BuChE activity for the method. Coming about, plan, and synthesis of double $\mathrm{AChE}$ or BuChE inhibitors ought to be considered to distinguish increasingly powerful specialists for treating AD. A few dynamic mixes by joining $\mathrm{AChE}$ and $\mathrm{BuChE}$ inhibitors into a solitary particle have gained critical ground (Bezerra, 2016). In this review, we talk about the current status in the treatment of Alzheimer's ailment just as future difficulties in the clinical application for Alzheimer's malady.

\section{FDA Approved AD medications:}

Drug treatment is a vital clinical strategy. It mainly delays neuronal degeneration and is mainly of therapeutic benefit only in patients with early-onset AD. The research and development of anti-AD drugs or antibodies mainly focus on three targets:

1) Against-oxidation,

2) Expulsion of $A \beta$ stores in the cerebrum,

3)Regulation of tau protein phosphorylation (Godyn, 2016).

At present, clinical treatment using anti-AD drugs mainly involve the use of acetylcholinesterase inhibitors to improve cognitive ability. In which three cholinesterase inhibitor's (CIs) and N-methylD-aspartate (NMDA) receptor antagonists, such as memantine, are at present accessible and have been endorsed for the therapy of middling $\mathrm{AD}$ but none of these agents can cure the condition (Coley, 2015; Zhang ,2019). Memantine was the first NMDA (Nmethyl-D-aspartate) receptor antagonist endorsed in the United States and is recommended to improve perception, consideration, thinking, language, and the capacity to perform straightforward errands in unremarkable Alzheimer's disease. In 2005, the FDA declined to extend the extent of memantine to mellow Alzheimer's malady, because of reactions, for example, cerebral pain, constipation, disarray, and tipsiness. In contrast to the next endorsed specialists, rivastigmine isn't used in the liver and, thus, doesn't incite the unfriendly medication associations of different medications generally recommended to older Alzheimer's disease patients as well as Tacrine also inhibits both the enzymes acetylcholinesterase and butyryl-cholinesterase but due to serious hepatic side reactions, it was prohibited from market. Galantamine exhibits low acetylcholinesterase selectivity, and its most common reactions are nausea and vomiting, which are normally self-restricting. In contrast to the next affirmed operators, it likewise regulate the action of nicotinic receptor. Donepezil has barely genuine sde reactions such as looseness of bowels and consequently it is utilized more oftentime than different referenced. The blend of donepezil and memantine has likewise been affirmed by the Food Drug Administration for the treatment of moderateto-outrageous Alzheimer's illness in patients who have been taking donepezil hydrochloride $(10 \mathrm{mg})$ (Atri , 2019). However, no new medication has been endorsed by the Food Drug Administration since 2003 despite many long and expensive trials (Hukins et al. 2019). More than 200 research projects in the last decade have failed or have been abandoned (Atri, 2019).

\section{Nanotechnology in AD therapy}

Nanotechnological advancement has the potential to give effective diagnostic and therapeutic options. The delivery of targeted drug with the help of nanoparticles can efficaciously cross the bloodbrain barrier with least side effects. Shadab et al. 2018 announced that a naringenin nanoemulsion is a flavonoid inferred tranquilize that can secure the neurons against free radicals and aggravation. It might likewise lessen the neurotoxic impacts of beta-amyloid plaque and phosphorylated tau protein levels in the cell line, yet has restricted capacity to cross biomembranes (Shadab, 2018). Patel et al. 2007 arranged sialic corrosive conjugated dendrimers and remarked that they emulated the sialic corrosive found in the cell surface and decreased the neurotoxic impact of $\beta$-amyloid in plaque. They reasoned that such operators may be valuable for forestalling the harmful impacts of $\beta$ amyloid plaque in Alzheimer's disease (Patel, 2007). 


\section{Anti-inflammatory effects on Alzheimer's disease}

Although the neuroinflammation approach has been proposed as a possible mechanism for the pathogenesis of Alzheimer's. Epidemiological evidence suggests that continuing utilization of NSAIDs protects against the advancement of Alzheimer's disease. Regardless of this reason, imminent examinations indicated an absence of viability (Aisen et al. 2002. 2003). Only a couple of studies have endeavored to determine the epidemiology of Alzheimer's disease.

\section{Stem Cell Therapy}

Recently, a novel technique, stem cell therapy, has shown great potential in treating AD patients. An online search of the PubMed database was performed for articles and reviews published from 1998-2018 with the terms "Alzheimer's disease", "stem cells therapy", "neural stem cells". Astrostem is a stem cell-based treatment, derived from autologous adipose tissue. It is currently estimated in phase II study while human mesenchymal stem cell treatment is evaluated in a phase I study (Cummings, 2019).

However direct implantation of neural stem cells is not attainable because of their limited sources and compulsive immunogenicity (Zhang, 2020).

\section{Alzheimer's Challenges in the Future and Emerging trends:}

Until compelling treatment is accessible for $\mathrm{AD}$, researcher must give full endeavors to distinguish mediation that help commitment of patients to ensure the quality of life. (Kortte, 2013). However, outcomes of Alzheimer's treatment are not so are not all that great regardless of many research endeavors Nanotechnology appears to be an innovative tool for advanced research by modulating the pathway in targeted locations. This approach can end up providing a remarkable breakthrough in early-stage therapy of Alzheimer's disease. The most efficient AD therapy will target many criteria of Alzheimer's disease pathophysiological cascade from the hyperphosphorylation of tau protein, combine with neuroprotective factors. A new drug Cerebrolysin $\mathrm{CBL}$, that is a brain-derived peptide preparation has been reported in the model of Alzheimer's disease and human clinical trials. CBL consists of a small peptide chain with a neurotrophic activity that is thought to be involved in the cerebrolysin (Viegas, 2011) which is a peptide-based medication item, supporting the soundness, and the capacity of Neurons. It diminishes the Amyloid creation and improves intellectual and social exhibitions.

Cerebrolysin has also been shown a huge impact on the modulation of GSK3, and CDK5 activity results decline in the levels of tau phosphorylation (Fan, 2014). Additionally, an ongoing preliminary of Cerebrolysin was appeared to improve the exercises of day by day living and mental shortages in patients with mellow to direct extreme AD. But it may be of essential centrality to all the more likely comprehend the connection between tau, $A \beta$, and different components to create prosperous illness altering drugs (Galimberti and Scarpini, 2011).

The systems basic the pathogenesis of AD should be altogether researched before concentrating on the advancement of novel ailment changing mixes. In spite of promising premises identified with various pathogenic systems, enormous stage III preliminaries with conceivably ailment adjusting properties have neglected to exhibit any impact on discernment.But it may be overcome by the development of the new biocompatible molecule.

Considering the several advantages of stem cell therapy, more focus should be paid to a scientific \& medical research health system but there are still so many quandaries to be solved before they can be elongated to clinical applications as well as the development of multi target-directed ligands is an emerging strategy for improving the quality of treatment against a complex disease like Alzheimer disease.

\section{Conclusion}

Recently, a noteworthy progress has been done in comprehension to the genetic cause and the molecular basis of Alzheimer disease. However, more research is needed to further explore the action of medications in seeking a promising therapy for $\mathrm{AD}$. The toxicity issue is observed as a major challenge to nanomedicines but we can believe that it may overcome by design \& development of more biocompatible nanoparticles. Computer Aided Drug Design has been widely inspected for growing new medication to improve the achievement pace of medication discovery. In the Future, more CADD methods will be analyzed 


\section{Khare and Fatima}

to distinguish potential Anti-Alzheimer's medication. Though, more research is required to explore for further investigation. Another more foucus is being paid to stem cell therapy that may assume a key job in the treatment of Alzheimer's sickness. Although there are many unexplained things are identified with the utilization of undifferentiated cell with more challenges, yet it is still prospective method for the treatment of $\mathrm{AD}$. With above all attempts, it can be consider that effective treatment for $\mathrm{AD}$ will be available soon.

\section{References}

Aisen, P., Schafer, K., Grundman, M., Pfeiffer, E., Sano, M., Davis, K. 2003. Effects of rofecoxib or naproxen vs placebo on Alzheimer's disease progression: a randomized controlled trial. Journal of the American Medical Association, 289: 2819-2826.

Aisen, P., Schmeidler, J. and Pasinetti, G. 2002. Randomized pilot study of nimesulide treatment in Alzheimer's disease. Neurology, 58:1050-1054.

Atri A. 2019. Current \& Future Treatments in Alzheimer's disease. Semin Neurol, 39:227-240.

Barbosa M., Valentino P., Andrade P.B. 2014. Bioactive compounds from macroalgae in the new millennium: implications for neurodegenerative diseases. Mar. Drugs, 12(9):4934-4972.

Bezerra da Silva, C. and Pott, A. 2016. Effect of Donepezil, Tacrine, Galantamine, and Rivastigmine on acetylcholinesterase inhibition in Dugesia tigrina. Molecules,21(1):53-55.

Crismon, M.L. 1994. Tacrine: first drug approved for Alzheimer's disease. Ann. Pharmacother., 28(6):744-751.

Cummings J, Lee G, Ritter A, Sabbagh M. and Zhong K. 2019. Alzheimer's disease drug development pipeline: 2019 Alzheimers Dement., 5: 272-293.

Coley N, Gallini A. and Andrieu S. 2015. Prevention studies in Alzheimer's disease: progress towards the development of new therapeutics. CNS Drugs, 29:519-528.

Ernst,R.L. and Hay,J.W. 1997. Economic research on Alzheimer's disease: a review of the literature. Alzheimer Dis. Assoc. Disord., 11(6):135-145.
Fan, L.Y. and Chiu, M.J. 2014. Combotherapy, and current concepts as well as future strategies for the treatment of Alzheimer's disease. Neuropsychiatr. Dis. Treat., 10(10): 439-451.

Godyn, J., Jonczyk, J., Panek, D. and Malawska, B. 2016. Therapeutic strategies for Alzheimer's disease in clinical trials. Pharmacol Rep, 68: 127-138.

Galimberti, D. and Scarpini, E. 2011. Disease-modifying treatments for Alzheimer's disease. Ther Adv Neurol Disord, 4: 203-216.

Hukins, D., Macleod, U. and Boland, J.W. 2019. Identifying potentially inappropriate prescribing in older people with dementia: a systematic review. Eur J Clin Pharmacol.,75: 467-481.

Kortte, K.B. and Rogalski, E.J. 2013. Behavioral interventions for enhancing life participation in behavioral variant frontotemporal dementia and primary progressive aphasia. Int Rev Psychiatry., 25(2): 237-245.

Shadab, Md 2018. In vitro neuroprotective effects of naringenin nanoemulsion against $\beta$-amyloid toxicity through the regulation of amyloidogenesis \& tau phosphorylation. Int J.Boil Macromol,118: 1211-1219.

Patel, D.A. 2007. Attenuation of b-amyloid- induced toxicity by sialic acid conjugated dendrimers: role of sialic acid attachment. Brain Res, 1161: 95-105.

Viegas, F.P.D., Simoes, M.C.R., Rocha, M.D., Castelli, M.R., Moreira, M.S. and Junior, C.V., 2011. Alzheimer's Disease: characterization, evolution, and implications of the neuroinflammatory process. Rev. Virtual Quim., 3(4): 286-306.

Zhang, L., Liu, J.J., Zhao, Y., Liu, Y. and Lin, J.W. 2019. Nbutylphthalide affects the cognitive function of APP/PS1 transgenic mice (Alzheimer's disease model). Chinese Journal of Tissue Engineering Research, 19: 3025-3030.

Zhang, F.Q., Jiang, J.L., Zhang, J.T., Niu, H., Fu, X.Q., Zeng, L.L. 2020. Current status and future prospects of stem cell therapy in Alzheimer's disease. Neural Regen Res, 15:242-250. 